\title{
Micro-tearing modes in spherical and conventional tokamaks
}

\author{
S. Moradi ${ }^{1}$, I. Pusztai ${ }^{1,3}$, W. Guttenfelder ${ }^{2}$, T. Fülöp ${ }^{1}$ and A. Mollén ${ }^{1}$ \\ ${ }^{1}$ Department of Applied Physics, Nuclear Engineering, \\ Chalmers University of Technology and Euratom-VR Association, Göteborg, Sweden \\ 2 Princeton Plasma Physics Laboratory, Princeton NJ 08543, USA \\ ${ }^{3}$ Plasma Science and Fusion Center, Massachusetts \\ Institute of Technology, Cambridge MA, 02139, USA
}

\begin{abstract}
The onset and characteristics of Micro-Tearing Modes (MTM) in the core of spherical (NSTX) and conventional tokamaks (ASDEX-UG and JET) are studied through local linear gyrokinetic simulations with GYRO [J. Candy and E. Belli, General Atomics Report GA-A26818 (2011)]. For experimentally relevant core plasma parameters in the NSTX and ASDEX-UG tokamaks, in agreement with previous works, we find MTMs as the dominant linear instability. Also, for JET-like core parameters considered in our study an MTM is found as the most unstable mode. In all these plasmas, finite collisionality is needed for MTMs to become unstable and the electron temperature gradient is found to be the fundamental drive. However, a significant difference is observed in the dependence of linear growth rate of MTMs on electron temperature gradient. While it varies weakly and non-monotonically in JET and ASDEX-UG plasmas, in NSTX it increases with the electron temperature gradient.
\end{abstract}

\section{INTRODUCTION}

In recent years, specially in view of an increasing interest in high $\beta_{e}$ operation scenarios, such as hybrid scenarios for ITER [1, 2], the impact of electromagnetic effects on the particle and heat transport has attracted much attention. Here $\beta_{e}=\left(8 \pi n_{e} T_{e}\right) / B_{u n i t}^{2}$, where $n_{e}$ and $T_{e}$ are the electron density and temperature. $B_{\text {unit }}$ is defined as the effective field strength [3].

Recent reports have shown the significant role of electromagnetic modes such as MicroTearing Modes (MTMs) on the electron heat transport in the core of fusion plasmas [4 7]. It has been found that in plasmas where $\beta_{e}$ and collisionality are sufficiently high MTMs 
can become the dominant instability. Under these conditions, MTMs generate a major contribution to the anomalous electron heat flux.

The MTMs are small-scale in the radial direction, but ion-scale in the binormal direction. They are electromagnetic modes with an even parity with respect to the perturbed parallel vector potential, $\delta A_{\|}$(referred to as tearing parity). In the literature two drive mechanisms for MTMs are proposed: one is the time-dependent thermal force experienced by electrons which results in a parallel current that produces magnetic field perturbations. If these perturbations then tip the field lines in the direction of an equilibrium electron temperature gradient the thermal force will increase and therefore the instability arises [8]. The second mechanism is due to a current carried by nearly trapped electrons in a boundary layer close to the trapped-passing boundary [9, 10]. Both of these mechanisms require finite electron temperature gradient and collisions. However, MTMs have been observed to arise under conditions which are not well-described by the above mechanisms [4, 11]. Thus, a complete picture of MTM excitation is not available at present.

In the following we summarize the previous findings reported in Refs. [4, 11 14]. Linear simulations for MAST [4, 11], NSTX [12], and ASDEX-UG [14] plasmas reported that MTMs can be the dominant instabilities in the region $r / a=0.5-0.8$ with the maximum growth rate at mode numbers $k_{\theta} \rho_{s}$ between $0.2-0.8$. Here, $\rho_{s}$ is the ion sound Larmor radius. The electron temperature gradient is found to be the drive of the instability and a non-monotonic dependence of the growth rate on the electron-ion collisionality $\nu_{e i}$ is observed. The peak of the growth rate coincides with the experimental value of $\nu_{e i}$ for various considered radial positions. The non-monotonic dependence of the MTM growth rate on collisionality is due to the fact that, on one hand both of the driving mechanisms mentioned above require finite collisionality, and therefore stability can be expected as $\nu_{e i}$ reduces, but on the other hand, in a strongly collisional regime the strong rate of scattering of the electrons between the field lines prevents the formation of a current layer, hence MTMs are stabilized [14].

It has also been shown that by increasing the effective ion charge $Z_{\text {eff }}$, MTMs are destabilized through the $Z_{e f f}$-dependence of the electron-ion collision frequency, see Ref. [12]. As MTMs are electromagnetic in nature a finite $\beta_{e}$ is needed for their destabilization. For the studied discharge in Ref. [12] it is found that the $\beta_{e}$ threshold is well below the experimentally relevant $\beta_{e}$ value and the growth rate increases moderately with increasing $\beta_{e}$. 
Non-linear simulations confirmed the role of the electron temperature gradient as the drive of the MTM instability in spherical (NSTX) [5] as well as standard tokamaks (ASDEXUG) [14]. Moreover, it has been shown that the Chirikov criterion [15] for overlapping of the magnetic islands leads to an up-shift of the electron temperature gradient threshold [5, 6, 13, 14].

In the present paper we investigate the onset of the MTMs and its parametric dependence through local linear gyrokinetic simulations with the GYRO code [16], in a spherical tokamak: NSTX, and two conventional tokamaks: ASDEX-UG and JET. For the NSTX case we use the plasma parameters reported in Ref. [12, 13], and for the ASDEX-UG case we use the plasma parameters found in Ref. [14]. In the present work we re-examine these discharges with an emphasis on the parametric dependences of the MTM onset. In view of the new ITER-like wall experiments on JET, in the presented analysis we have also considered a set of JET-like parameters.

The remainder of the paper is organized as follows. In Sec. II the input parameters are discussed, and in Sec. III) parametric dependences of the MTM onset are analyzed by presenting scans over MTM driving parameters such as collisionality, $\beta_{e}$ and electron temperature/density scaling lengths. The conclusions are drawn in Sec. IV]

\section{INPUT PARAMETERS}

The plasma parameters used in our analysis are shown in table I.

TABLE I: Input parameters for densities, temperatures and their gradients.

\begin{tabular}{|c|c|c|c|c|c|c|c|c|c|c|}
\hline \hline & $r / a$ & $Z_{\text {eff }}$ & $n_{e}\left[10^{19} / m^{3}\right]$ & $T_{e}[k e V]$ & $a / L_{n e}$ & $a / L_{T i}$ & $a / L_{T e}$ & $T_{i} / T_{e}$ & $\rho_{s} / a$ & $\nu_{e i}\left(a / c_{s}\right)$ \\
\hline NSTX & 0.6 & 2.91 & 6.0 & 0.44 & -0.83 & 2.36 & 2.72 & 0.94 & 0.0074 & 1.45 \\
\hline AUG & 0.65 & 3.30 & 7.6 & 0.765 & 0.37 & 2.18 & 3.02 & 1.19 & 0.0018 & 0.68 \\
\hline JET & 0.6 & 3.41 & 7.8 & 1.25 & 0.15 & 2.16 & 2.16 & 1.00 & 0.0027 & 0.43 \\
\hline
\end{tabular}

Here, $L_{n}=-[\partial(\ln n) / \partial r]^{-1}, L_{T}=-[\partial(\ln T) / \partial r]^{-1}$, are the density and temperature scale lengths, $a$ is the outermost minor radius. Deuterium ions, an active impurity species denoted in table $\amalg$ by $Z_{1}$ (carbon for NSTX and nitrogen for ASDEX-UG and JET), and a passive species of impurity (tungsten unless otherwise stated) denoted in the table $\amalg$ by $Z_{2}$, 
TABLE II: Input parameters for plasma ion compositions.

\begin{tabular}{|c|c|c|c|c|c|}
\hline \hline & $Z_{1}$ & $n_{Z 1} / n_{e}$ & $a / L_{n Z 1}$ & $Z_{2}$ & $n_{Z 2} / n_{e}$ \\
\hline NSTX & $C^{+6}$ & $6.4 \%$ & -2.75 & $W^{+40}$ & $0.02 \%$ \\
\hline AUG & $N^{+7}$ & $4.8 \%$ & 0.80 & $W^{+40}$ & $0.02 \%$ \\
\hline JET & $N^{+7}$ & $5.0 \%$ & 0.14 & $W^{+40}$ & $0.02 \%$ \\
\hline
\end{tabular}

are considered. The passive species are considered here in order to examine the impurity particle transport due to MTMs. Note that in the ASDEX-UG case reported in Ref. [14] no impurities were present and the value of $a / L_{T i}$ has been artificially reduced from its experimental value to eliminate the drive of Ion Temperature Gradient (ITG) modes, but here we use the experimental values.

Linear runs with GYRO include full electromagnetic effects: shear $\delta B\left(=\nabla \times \delta A_{\|}\right)$, and compressional $\delta B_{\|}$magnetic perturbations. Gyrokinetic electrons are assumed, and the collisions are modeled using an energy dependent Lorentz operator. Both electron-ion and electron-electron collisions are included in the electron collision frequency $\nu_{e}(v)$, and collisions between all ion species are accounted for. To take into account the plasma shape we have used a Miller-type local equilibrium model available in GYRO, see Refs. [3, 17]. Typical JET parameters for plasma shape and magnetic geometry are used, and the corresponding values are given in Table III. In this table, $\beta_{e}$ is calculated in CGS units following the

TABLE III: Input parameters for plasma shape and magnetic geometry.

\begin{tabular}{|c|c|c|c|c|c|c|c|c|c|c|c|}
\hline \hline & $a[m]$ & $\beta_{e}$ & $\alpha_{M H D}$ & $B_{\text {unit }}$ & $R_{0} / a$ & $B_{0}[T]$ & $s$ & $q$ & $\kappa$ & $\delta$ & $k_{\theta} \rho_{s}$ \\
\hline NSTX & 0.6 & 0.024 & 0.36 & 0.66 & 1.52 & 0.35 & 1.73 & 1.68 & 1.72 & 0.12 & 0.63 \\
\hline AUG & 0.6 & 0.005 & 0.42 & 2.16 & 3.3 & 2.479 & 1.31 & 2.18 & 1.30 & 0.13 & 0.2 \\
\hline JET & 1.0 & 0.013 & 0.35 & 1.77 & 3.3 & 2.55 & 1.32 & 1.45 & 1.70 & 0.37 & 0.5 \\
\hline
\end{tabular}

expression:

$$
\beta_{e}=\frac{8 \pi\left(n_{e}\left[10^{19} / m^{3}\right] 10^{-6} 10^{19}\right)\left(T_{e}[k e V] 1.602210^{-9}\right)}{\left(10^{4} B_{\text {unit }}[T]\right)^{2}},
$$

where $B_{\text {unit }}=\left(d \chi_{t} / d r\right) / r$ is the effective field strength with $\chi_{t}$ defined through the toroidal magnetic flux $2 \pi \chi_{t}[3,17], q$ is the safety factor, and the magnetic shear is $s=(r / q) d q / d r$. 
The generalized magnetohydrodynamic $\alpha$ parameter is defined as

$$
\alpha_{M H D}=-q^{2} R_{0} \frac{8 \pi}{B_{\text {unit }}^{2}} \frac{d p}{d r} c_{p},
$$

where $R_{0}$ is the effective major radius, $r$ is the minor radius, and $p=\sum_{a} n_{a} T_{a}$ is the total plasma pressure. $c_{p}$ is a scaling parameter which allows an artificial adjustment of $\alpha_{M H D}$ (affecting the magnetic curvature drift) without modifying the background gradients, as presented in Ref. [18]. Furthermore, $\kappa$ is the elongation, $\delta$ is the triangularity.

Typical resolution parameters used in our linear analysis are as follows: 40 radial grid points, 12 parallel orbit mesh points ( $\times 2$ signs of parallel velocity), 16 pitch angles, and 8 energies. A high radial resolution is needed since the linear instability of MTM depends on the presence of narrow resonant current layers centered on the rational surfaces. The toroidal mode numbers used here, corresponding to the $\rho_{s} / a$ values in table I, are $n=30$ for NSTX, $n=18$ for AUG, and $n=75$ for JET.

\section{MTM INSTABILITY}

Figures 1 $(a, b)$ illustrate the linear growth rate and the real frequency of the most unstable modes in the three machines as functions of $k_{\theta} \rho_{s}$. In NSTX and JET, the MTMs (with positive real frequency in electron diamagnetic direction) are the most unstable modes in the range of $0.1 \leq k_{\theta} \rho_{s} \leq 1$, while for ASDEX-UG the MTMs are the most unstable modes only in the narrower wave number range $0.1 \leq k_{\theta} \rho_{s} \leq 0.3$, corresponding to longer wavelengths than those in NSTX and JET. In NSTX the MTMs remain the most unstable mode for the whole considered range of $k_{\theta} \rho_{s}$, but in ASDEX-UG an ITG mode (negative real frequency, in ion diamagnetic direction) for $0.3<k_{\theta} \rho_{s}<1$ and an ETG mode (positive real frequency, in electron diamagnetic direction) for $1<k_{\theta} \rho_{s}$ are also found to be unstable. The $k_{\theta} \rho_{s}$ corresponding to the maximum growth rate varies between the different machines: in NSTX $k_{\theta} \rho_{s} \sim 0.6$, in ASDEX-UG $k_{\theta} \rho_{s} \sim 0.2$ and in the JET like case $k_{\theta} \rho_{s} \sim 0.5$. The normalized poloidal mode number for the remainder of our calculations are fixed to these values (see table III). Previous studies have discussed the difference in the poloidal mode number corresponding to the maximum of the unstable MTMs between the NSTX and ASDEX-UG cases, and it is believed to be due to characteristics of the spherical or

conventional tokamaks [12, 14]. However, here we find unstable MTMs with similar mode 

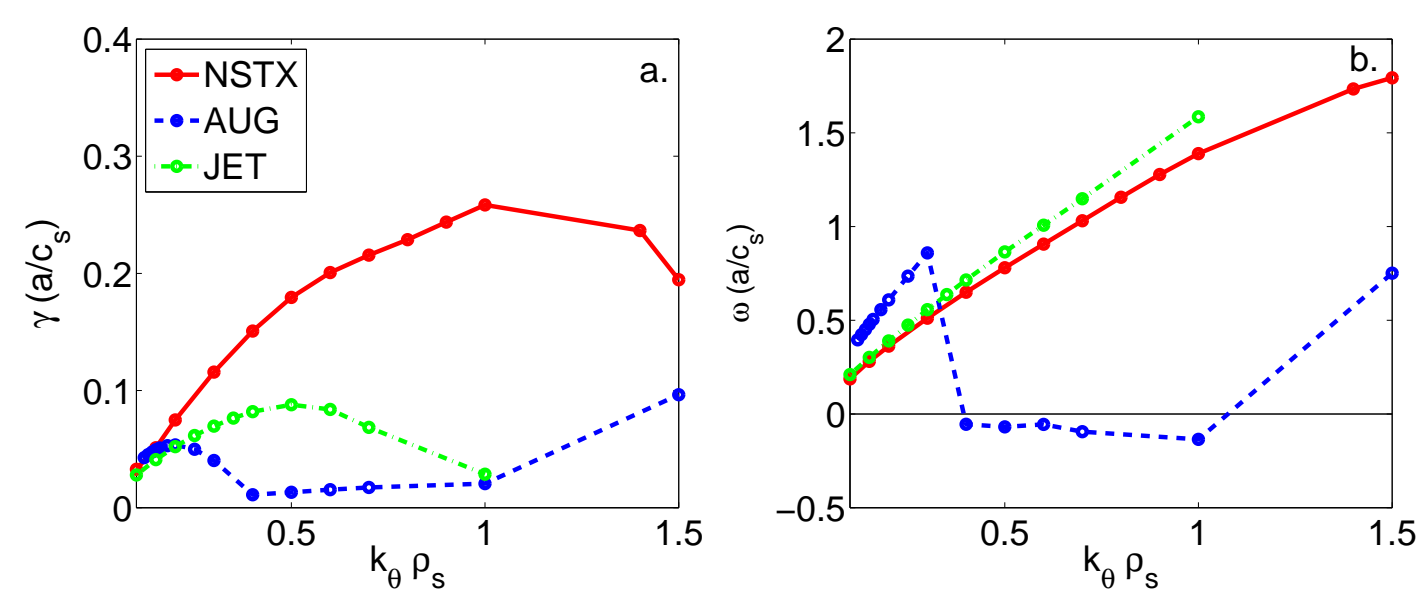

FIG. 1: Imaginary (a), and real parts (b) of eigenvalues $\left(\gamma, \omega_{r}\right)$ as functions of $k_{\theta} \rho_{s}$. Red solid lines: NSTX, blue dashed: ASDEX-UG, green dash-dotted: JET.

numbers in the JET tokamak to that of NSTX, which suggests that this is not always the case. Therefore, further studies are needed to determine the reason for the similarities and differences in the mode numbers for maximum growth rates in various machines.

The structure of the $\delta \phi, \delta A_{\|}$and $\delta B_{\|}$eigenmodes, corresponding to the $k_{\theta} \rho_{s}$ values mentioned above are shown in figures 2 (a-f). The eigenfunctions are normalized so that $\delta A_{\|}(\theta=0)$ is unity. The MTM signature is distinguished by the tearing parity of the $\delta A_{\|}$ eigenmodes. The NSTX case has the strongest electromagnetic character in terms of the relative amplitude of $\delta \phi$, while the "most electrostatic" mode is the AUG case. Regarding the strength of the compressional magnetic perturbations, $\delta B_{\|}$, the situation is the opposite; the less electrostatic the mode is the stronger the $\delta B_{\|}$perturbations are. The characteristic width of the eigenmodes along the field line is similar in all three machines.

Figures 3 and 4 illustrate the normalized linear energy and particle fluxes, respectively, and the contributions to these from the $\delta \phi, \delta A_{\|}$and $\delta B_{\|}$fluctuations, as functions of $k_{\theta} \rho_{s}$ in all three machines. As seen in figures $3(\mathrm{a}-\mathrm{c})$, in the MTM dominated region the electron heat flux is the main channel of the energy transport, and the dominant contribution is generated by $\delta A_{\|}$(blue dash-dotted line). In the NSTX case, see figure 3 (a), this is true for the whole considered range of $k_{\theta} \rho_{s}$. The ion heat flux generated by MTMs shown in figure 3 (d) (magenta solid line) however, is negligibly small compared to the electron heat flux. The particle fluxes generated by MTMs for electrons as seen in figure 4 (a-c), and both 

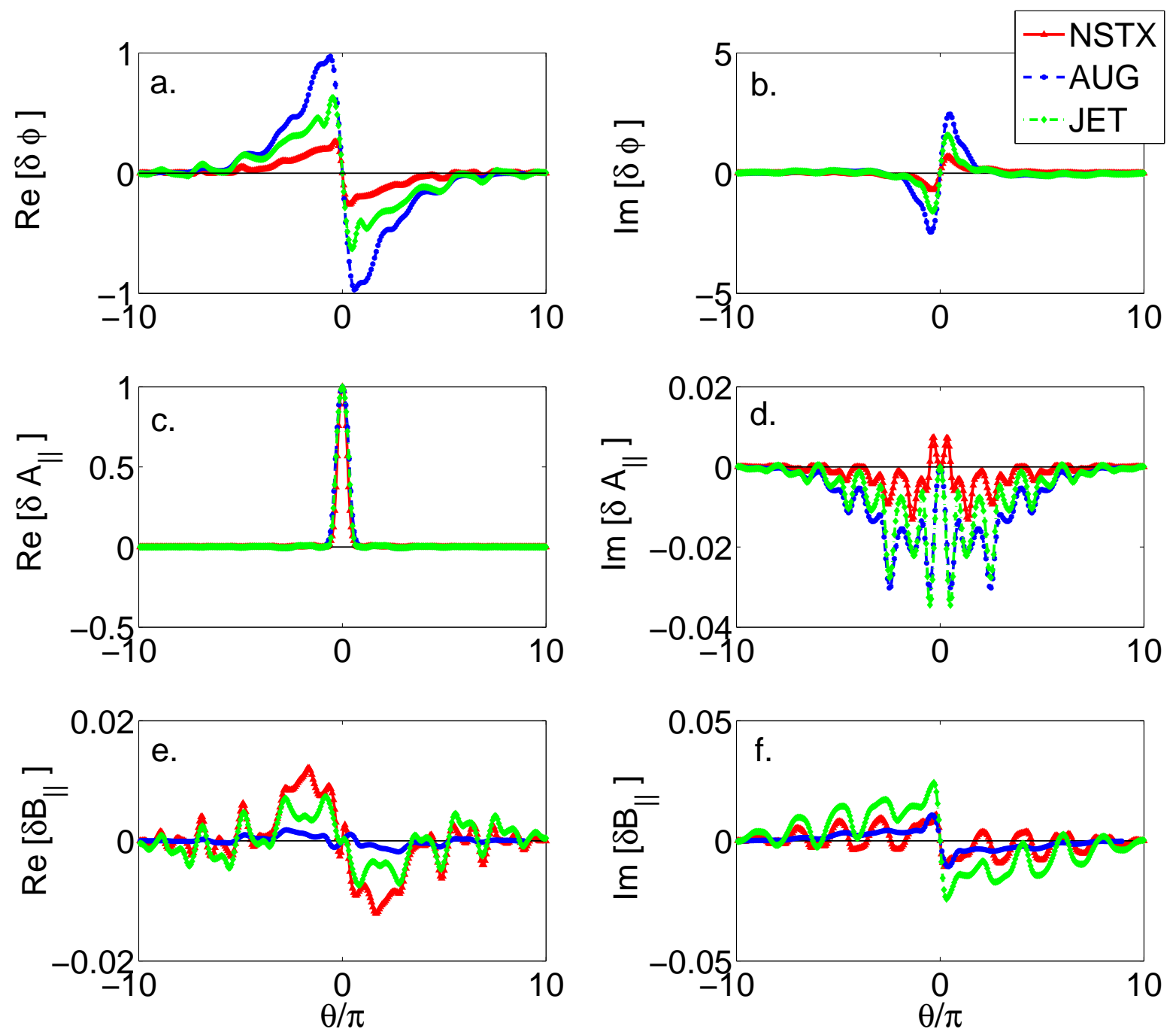

FIG. 2: Linear parallel mode structures of $\delta \phi(\mathrm{a}, \mathrm{b}), \delta A_{\|}(\mathrm{c}, \mathrm{d})$ and $\delta B_{\|}(\mathrm{e}, \mathrm{f})$. Note that the actual radial resolution of the simulations covers $\theta / \pi=(-16,16)$.

active and passive impurity species shown in figures 4 (d-f and g-i) are also negligible in comparison to the electron heat flux. We note, that nonlinear MTM simulations for NSTX, presented in [5, 13] also showed negligibly small particle fluxes.

In the case of ASDEX-UG the main contribution to electron heat flux, see figure $3(\mathrm{~b})$, is generated by the MTM instability at low $k_{\theta} \rho_{s}$, and for higher $k_{\theta} \rho_{s}$ where the most unstable mode switches to an ITG mode, the electron heat flux is significantly reduced. However, at the very high poloidal mode numbers $1<k_{\theta} \rho_{s}$ where an ETG is the dominant instability 

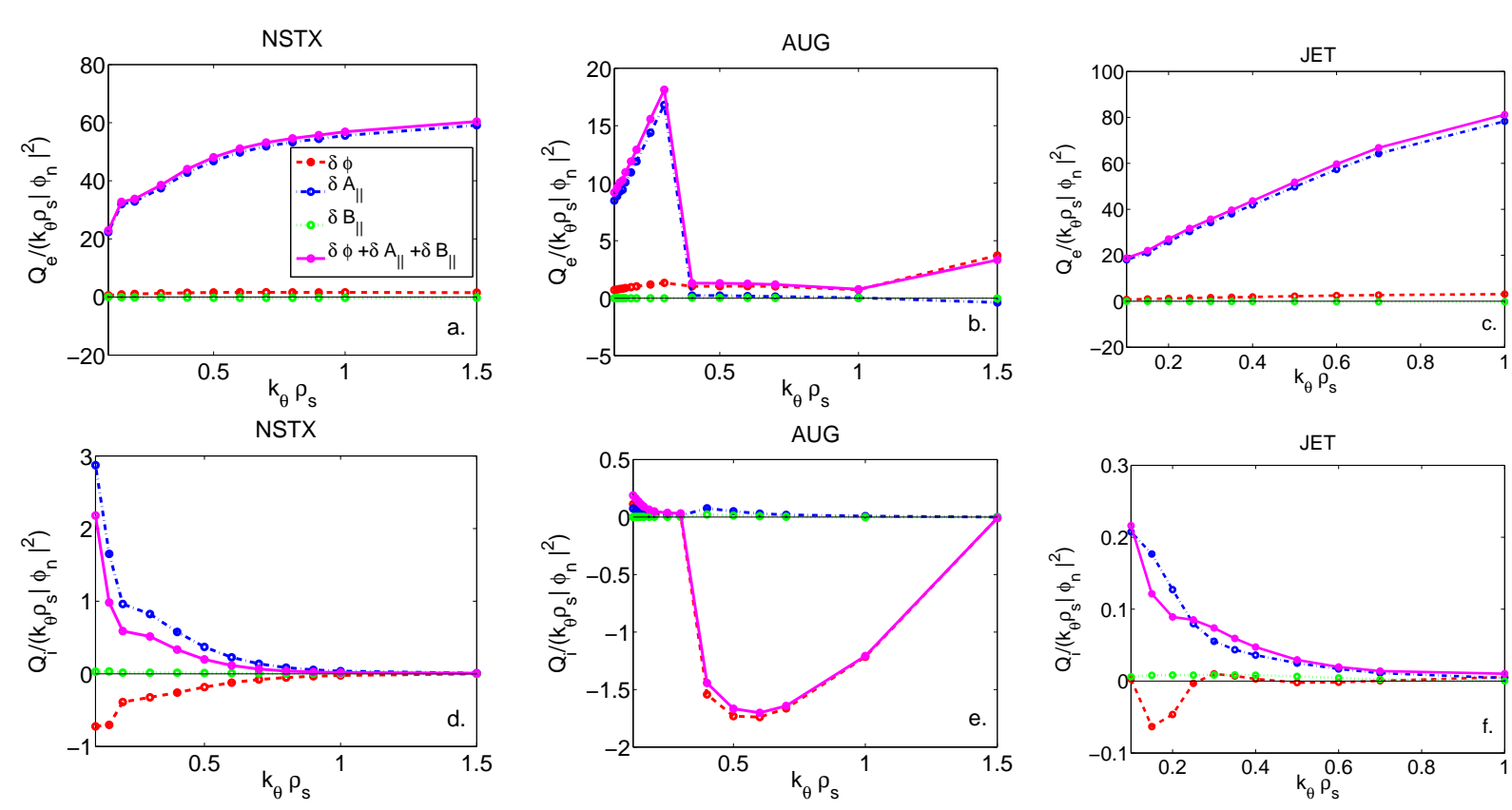

FIG. 3: Normalized linear electron (top) and ion (bottom) heat fluxes (magenta solid lines) and their contributions from $\delta \phi$ (red dashed lines), $\delta A_{\|}$(blue dash-dotted lines), and $\delta B_{\|}$(green dotted lines) versus $k_{\theta} \rho_{s}$ in NSTX (a, d), ASDEX-UG (b, e), and JET (c, f).

the electron heat flux increases again. Also here, $\delta A_{\|}$(blue dash-dotted line) generates the dominant contribution to the electron heat flux in the MTM dominated region while $\delta \phi$ (red dashed line) produces the dominant contribution to the electron heat flux in the ITG/ETG dominated regions. For the ion heat flux the main contribution comes from the higher $k_{\theta} \rho_{s}$ region where the ITG mode is the most unstable mode present with the maximum around $k_{\theta} \rho_{s} \sim 0.5$, as illustrated in figure 3 (e). The MTM and ETG contributions to the ion heat flux are significantly smaller. Also, for the electron and impurity particle fluxes, shown in figures 4 (b, e and h), the contributions from MTM and ETG is negligible compared to the contribution from the ITG.

Similar trends are observed for heat and particle transport in the JET case. Again here, in the MTM dominated region the electron heat flux, see figure 3 (c), is the main channel of transport while the ion heat flux, presented in figure 3 (f), and the particle fluxes shown in figures 4 (c, f and i) are negligibly small in comparison.

Remarkably, the main ion energy fluxes $\left(Q_{i}\right)$ generated by the ITG modes in the ASDEX$\mathrm{UG}$ is found to be inward in spite of the positive ion temperature gradient, see Figs. 3 (d). 

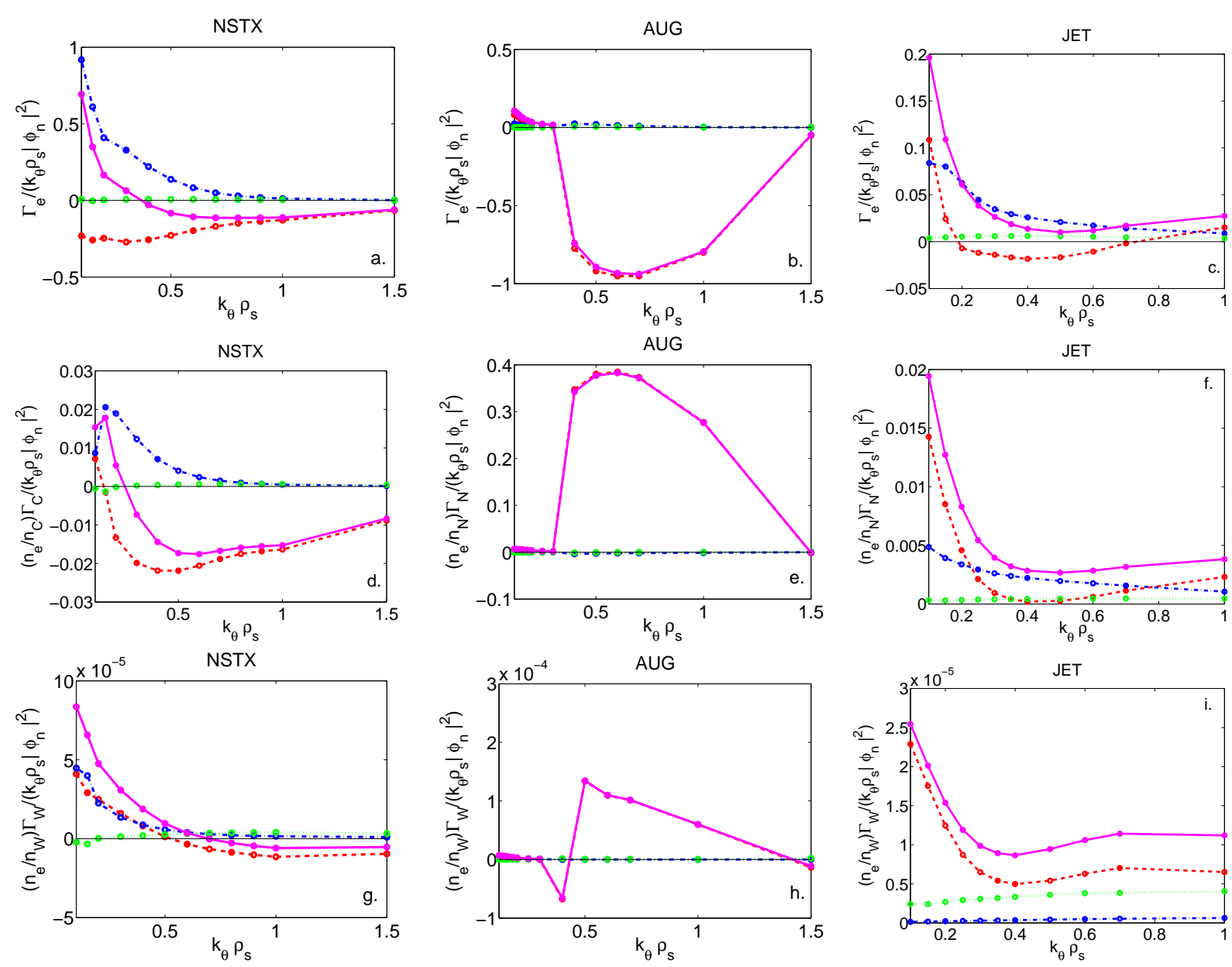

FIG. 4: Normalized linear particle fluxes (magenta solid lines) and their contributions from $\delta \phi$ (red dashed lines), $\delta A_{\|}$(blue dash-dotted lines), and $\delta B_{\|}$(green dotted lines) versus $k_{\theta} \rho_{s}$ in NSTX (a, $\mathrm{d}$ and $\mathrm{g}$ ), ASDEX-UG (b, e and h), and JET (c, f and i).

However we note, that in the ASDEX-UG case 1) there is a high inward particle flux of main ions which might account for the inward energy flux if most of it is convective, 2) the strong positive ion energy flux carried by the nitrogen impurities almost cancel the negative energy flux of the main ions, so the total ion energy flux is close to zero.

\section{A. Parametric dependences}

Figures 5 (a,c) show the linear growth rates and the real frequencies of the unstable modes, corresponding to the fixed values of $k_{\theta} \rho_{s}$ given in table III, as functions of $\beta_{e}$ for the three considered machines. As seen in this figure for the experimental values of $\beta_{e}$ (shown 
with vertical lines) the most unstable mode is an MTM for all three tokamaks. In NSTX, the onset of MTM is well below the experimental value of $\beta_{e}$ and an increase in $\beta_{e}$ above its experimental value does not increase the growth rate significantly. The same observation can be made for the JET case, while for ASDEX-UG, although the MTM onset is well below its experimental value, by further increasing $\beta_{e}$, a Kinetic-Ballooning Mode (KBM) (negative real frequency in ion direction) appears as the most unstable mode. These results show that since MTMs become unstable due to electromagnetic perturbations a finite level of $\beta_{e}$ is needed for their onset, but when unstable, MTMs do not strongly depend on $\beta_{e}$ variations. In all these scans the value of $\alpha_{M H D}$ is calculated consistently with the local beta values and the density and temperature gradients.
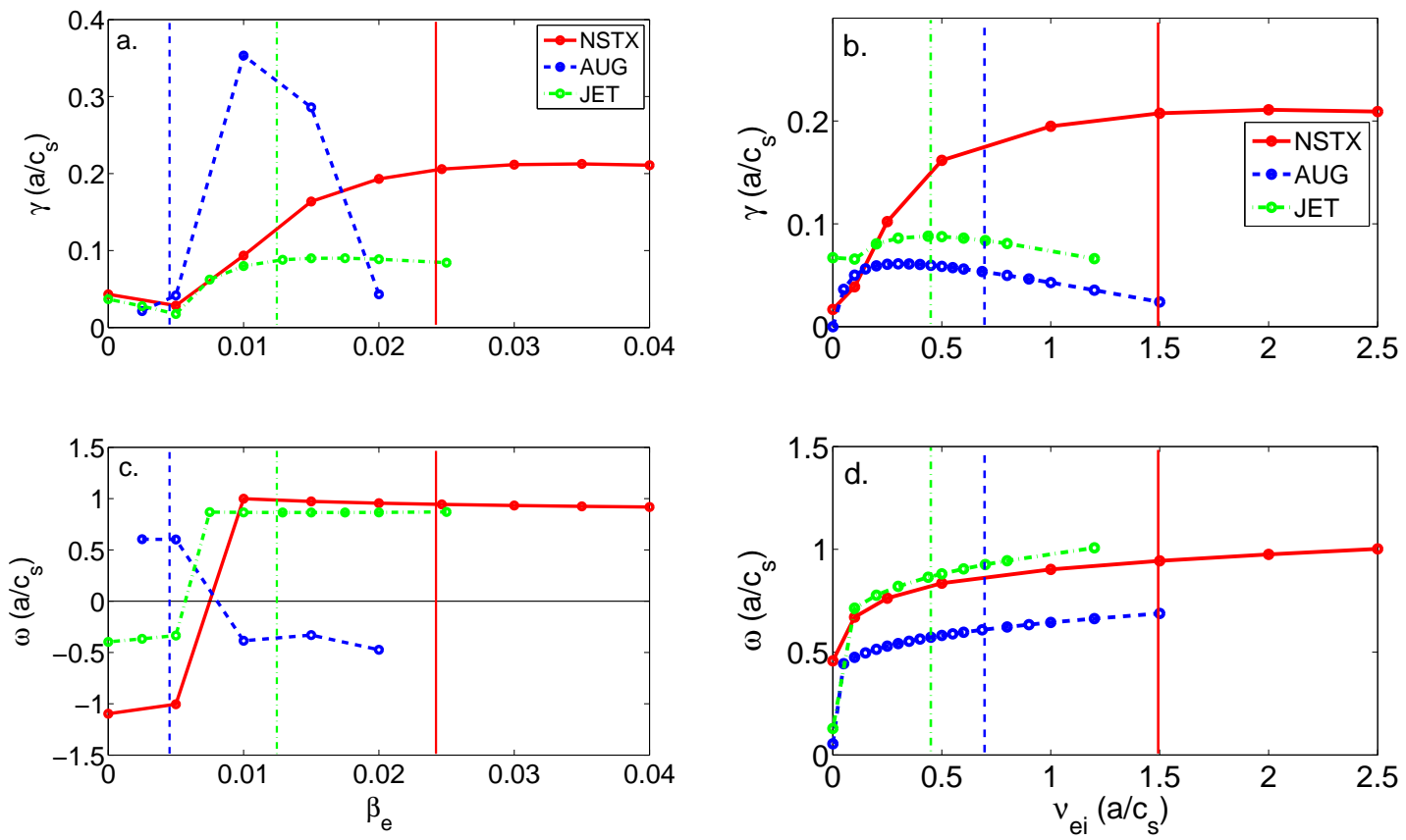

FIG. 5: Imaginary and real parts of eigenvalues $\left(\gamma, \omega_{r}\right)$ as functions of $\beta_{e}$ (a and c), and $\nu_{e i}$ (b and d). Red solid lines: NSTX, blue dashed: ASDEX-UG, green dash-dotted: JET. The vertical lines represent the base parameters color coded similarly to their respective machine.

In order to determine the effect of $\alpha_{M H D}$ through the curvature drift a $\beta_{e}$ scan, similar to figure 5 (a and c), is performed where $\alpha_{M H D}$ is scaled to zero by setting $c_{p}=0$. The corresponding results are shown in figure 6. The $\alpha_{M H D^{-}}$-stabilization is not significant in the MTM regime in any of the studied plasmas. In the NSTX case there is a small $\alpha_{M H D}$-stabilization 
only at very high $\beta_{e}$, see figures 6 (a,d). In the ASDEX-UG case, the stabilization of the KBM mode at higher $\beta_{e}$ is clearly an $\alpha_{M H D}$ effect, since without it the mode is further destabilized by an increase in $\beta_{e}$, see the black dashed lines in figures 6 (b,e). For JET, the situation is different, as seen in figures [6 (c,f). In the electrostatic limit and at low $\beta_{e}$ the most unstable mode is an ITG which is stabilized as $\beta_{e}$ is increased even without $\alpha_{M H D}$ effect. For higher $\beta_{e}$ the dominant mode switches to an MTM. Without $\alpha_{M H D}$ effects, by increasing $\beta_{e}$ even further the MTM switches to a KBM that is further destabilized by $\beta_{e}$, however with the $\alpha_{M H D}$ effect included the KBM appears only at higher $\beta_{e}$ (outside the plotted $\beta_{e}$ range).
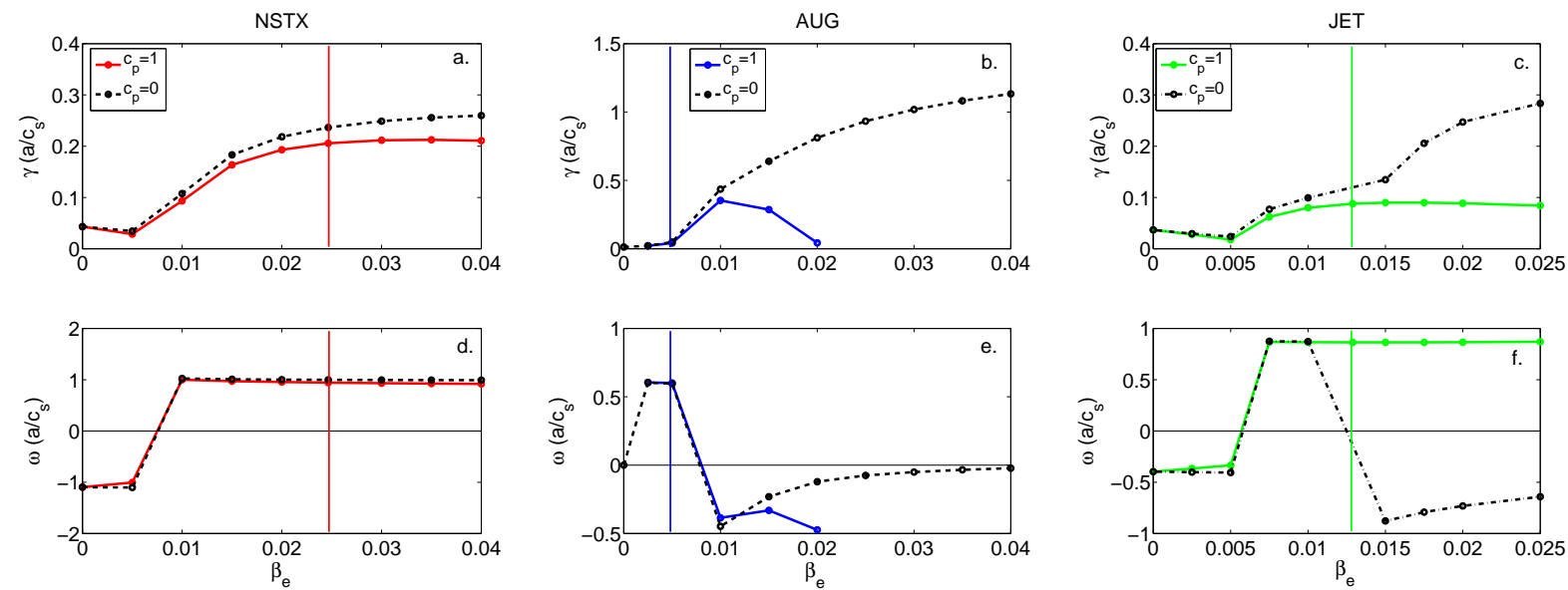

FIG. 6: Imaginary and real parts of eigenvalues $\left(\gamma, \omega_{r}\right)$ as functions of $\beta_{e}$ for $c_{p}=1$ (solid lines) and $c_{p}=0$ (dashed black lines). NSTX (a and d), ASDEX-UG (b and e), and JET (c and f). The vertical lines represent the base parameters color coded similarly to their respective machine.

As the collisionality is suggested as an important parameter in driving the MTMs unstable, here we examine the dependence of the MTM mode characteristics on this parameter. In figures $5(\mathrm{~b}, \mathrm{~d})$ the linear growth rates and the real frequencies of the most unstable modes are shown as functions of the collision frequency $\nu_{e i}$ for the different tokamaks. For the experimental values of $\nu_{e i}$ (marked with vertical lines) the most unstable mode is found to be an MTM in all the three considered tokamaks. In NSTX, the growth rate increases with collisionality as expected since collisionality is one of the instability drives [8, 9], however, a further increase in $\nu_{e i}$ above the experimental value (almost doubled) the MTM growth rate does not increase significantly. In the ASDEX-UG the growth rate shows a decline as 
collisionality increases. This trend is not surprising since previous studies have shown that the growth rate of the MTM has a non-monotonic dependence on the collisionality [4 6]. As the collisionality further increases beyond the peak value, particles are very much scattered by collisions, and therefore preventing the formation of the current layer necessary for the MTMs to become unstable. Hence, as seen in figure 5 (b) we expect that this is the case for the ASDEX-UG case. For JET we also observe a (gentle) non-monotonic trend, and our base value of the collisionality seems to be positioned near the peak value. In all three machines no unstable MTMs were found for the collisionless case, i.e. $\nu_{e i}=0$, and under this condition these plasmas are found to be TEM unstable. Using the eigenvalue solver method in GYRO [16] we followed the root corresponding to the MTM instability towards smaller $\nu_{e i}$, and the mode is completely stabilized in the collisionless limit. The values of $\nu_{e i} / \omega_{r}$ corresponding to the maximum growth rate are rather different between the spherical and the conventional tokamak cases; in NSTX it is 2.05, while it is 0.55 and 0.51 in the AUG and the JET cases, respectively.

In the literature the electron temperature gradient is suggested as one of the instability drives for MTMs [8, 9]. This has been confirmed in previous numerical studies [4-6]. Here we compare the role of electron temperature gradient in destabilization of the MTMs between the three considered machines by performing a scan over the $a / L_{T e}$ parameter. The results of this scan are shown in figures $7(\mathrm{a}, \mathrm{c})$ where the linear growth rates and the real frequencies of the most unstable modes are illustrated as functions of $a / L_{T e}$. As seen in this figure, a finite value of $a / L_{T e}$ is necessary for the destabilization of the MTMs in all three machines, however a clear difference is observed in the variation of MTM growth rates with $a / L_{T e}$ between spherical and conventional tokamaks. For the NSTX case, there is a clear increase of the MTM growth rate with an increase in $a / L_{T e}$, while for ASDEX-UG and JET the MTM growth rates show weaker and a non-monotonic dependence on $a / L_{T e}$. By further increase in $a / L_{T e}$ the most unstable mode switches from an MTM to ITG/TEM modes, corresponding to the last points in the ASDEX-UG and JET curves in Figs. 7 (a and c).

The $a / L_{T e}$ threshold for MTM instability is observed to be well below our baseline values (indicated by vertical lines), and it is lower for JET and ASDEX-UG than for NSTX. The growth rate of MTMs is found to be less sensitive to electron temperature gradient for both of the conventional tokamaks than in the spherical tokamak studied here. In order to investigate the reason for this difference we performed a similar scan over $a / L_{T e}$ and 

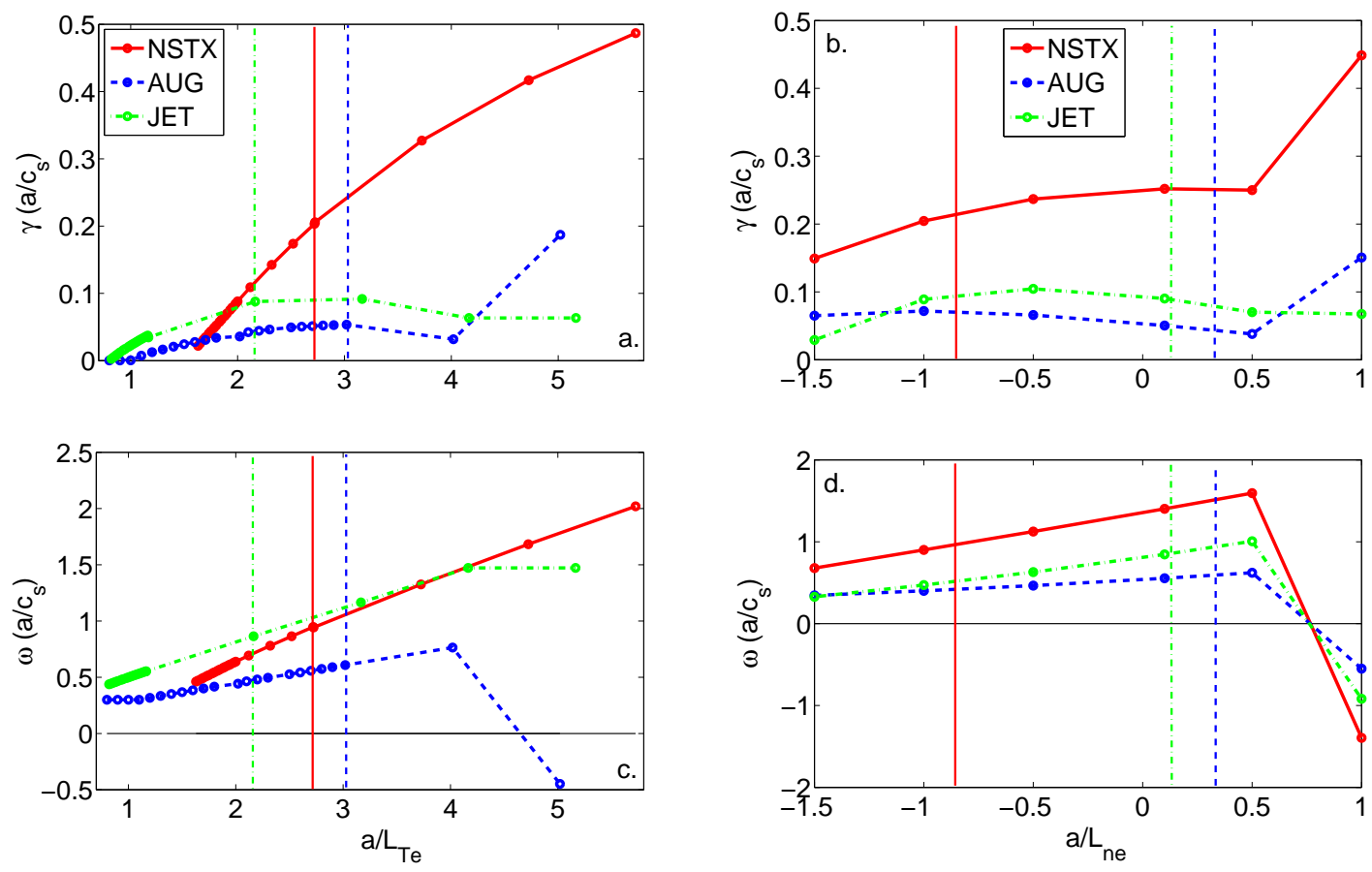

FIG. 7: Imaginary and real parts of eigenvalues $\left(\gamma, \omega_{r}\right)$ as functions of $a / L_{T e}$ (a and c), and $a / L_{n e}$ (b and d). Red solid lines: NSTX, blue dashed: ASDEX-UG, green dash-dotted: JET. The vertical lines represent the base parameters color coded similarly to their respective machine.

set $c_{p}=0$ to eliminate the $\alpha_{M H D}$ stabilization effect. The results are shown in figure 8 Without $\alpha_{M H D}$ stabilization the MTM still remains the most unstable mode for the baseline parameters, and as $a / L_{T e}$ increases no significant change is observed for NSTX and ASDEXUG. However, for JET case three regions in the $a / L_{T e}$ space can be distinguished. For low values of $a / L_{T e}$ the MTM is the dominant instability; its growth rate increases linearly with $a / L_{T e}$. At $a / L_{T e} \sim 2.5$ an ITG mode, which was previously stabilized by $\alpha_{M H D}$ effects see figures 7 (a,c), - becomes the dominant instability, which gradually transits to a TEM as $a / L_{T e}$ becomes very large. Therefore, from our observations the stabilizing effect of the $\alpha_{M H D}$ parameter on the MTM mode is not significant, but it has an impact on the stability of ITG/TEM/KBM modes. The strong suppression of these modes therefore, allows for MTMs to remain the dominant instability for a wider range of $a / L_{T e}$. The weaker and nonmonotonic dependence of the MTM on the electron temperature gradient in ASDEX-UG and JET thus, can not be explained by this effect.

One of the main differences between the plasma parameters in the three plasmas consid- 

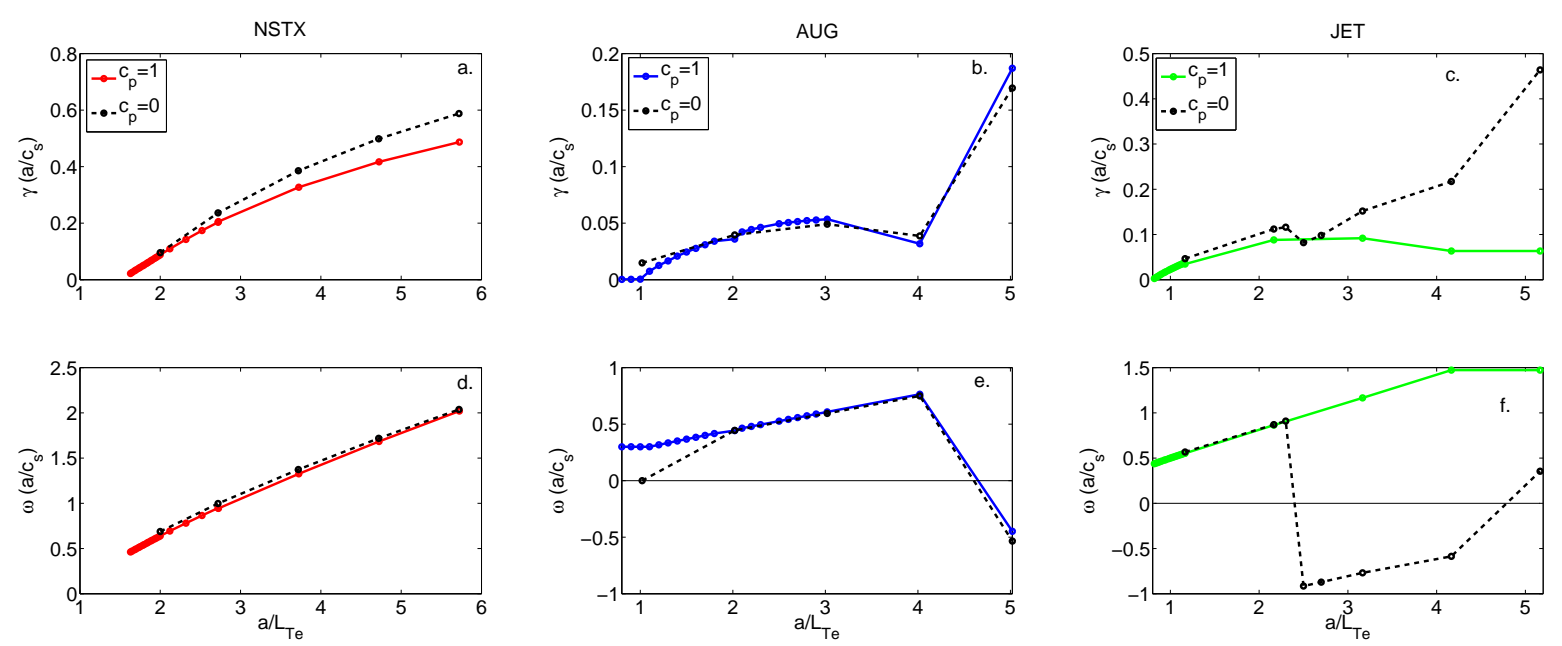

FIG. 8: Imaginary and real parts of eigenvalues $\left(\gamma, \omega_{r}\right)$ as functions of $a / L_{T e}$ with $c_{p}=1$ and $c_{p}=0$. Red solid lines: NSTX, blue dashed: ASDEX-UG, green dash-dotted: JET.

ered appears in the $a / L_{n e}$ values; a strongly negative electron density gradient, corresponding to a hollow electron density profile, is observed in NSTX, while the density profiles were slightly peaked in the ASDEX-UG and JET plasmas. Thus, we have investigated the dependence of MTM linear growth rates on the $a / L_{n e}$ parameter. Quasi-neutrality is enforced by slightly varying $a / L_{n i}$ while keeping the impurity density gradients fixed to the base parameters given in table $\llbracket$. Figures 7 (b-d) show the linear growth rates and the real frequencies of the most unstable modes versus $a / L_{n e}$ for the different machines. In all three machines, the MTMs linear growth rates exhibit a non-monotonic dependence on $a / L_{n e}$ parameter with maxima corresponding to slightly hollow electron density profiles $\left(a / L_{n e} \sim-0.5\right)$. Clearly $a / L_{n e}$ is not a strong and necessary drive for the MTMs, as there are finite MTM growth rates in all machines at $a / L_{n e}=0$.

In all the machines the MTM is the most unstable mode over the range of $-1.5 \leq$ $a / L_{n e} \leq 0.5$, with a rather weak dependence on this parameter. For sufficiently high electron density gradient the dominant linear mode switches from a MTM to an ITG mode in all plasmas. Again we examined the impact of $\alpha_{M H D}$ by comparing the results to corresponding simulations with $c_{p}=0$ (black dashed lines in figure 9). Similar to the results of the $a / L_{T e}$ scans shown in figure 8 , the effect of the $\alpha_{M H D}$ parameter on the MTM mode itself is negligible, but it strongly stabilizes the ITG mode. As shown in figure 9, without $\alpha_{M H D}$ 
effects in the cases of NSTX and JET the $a / L_{n e}$ threshold of the dominant ITG mode is reduced, but in ASDEX-UG case no significant change is observed.
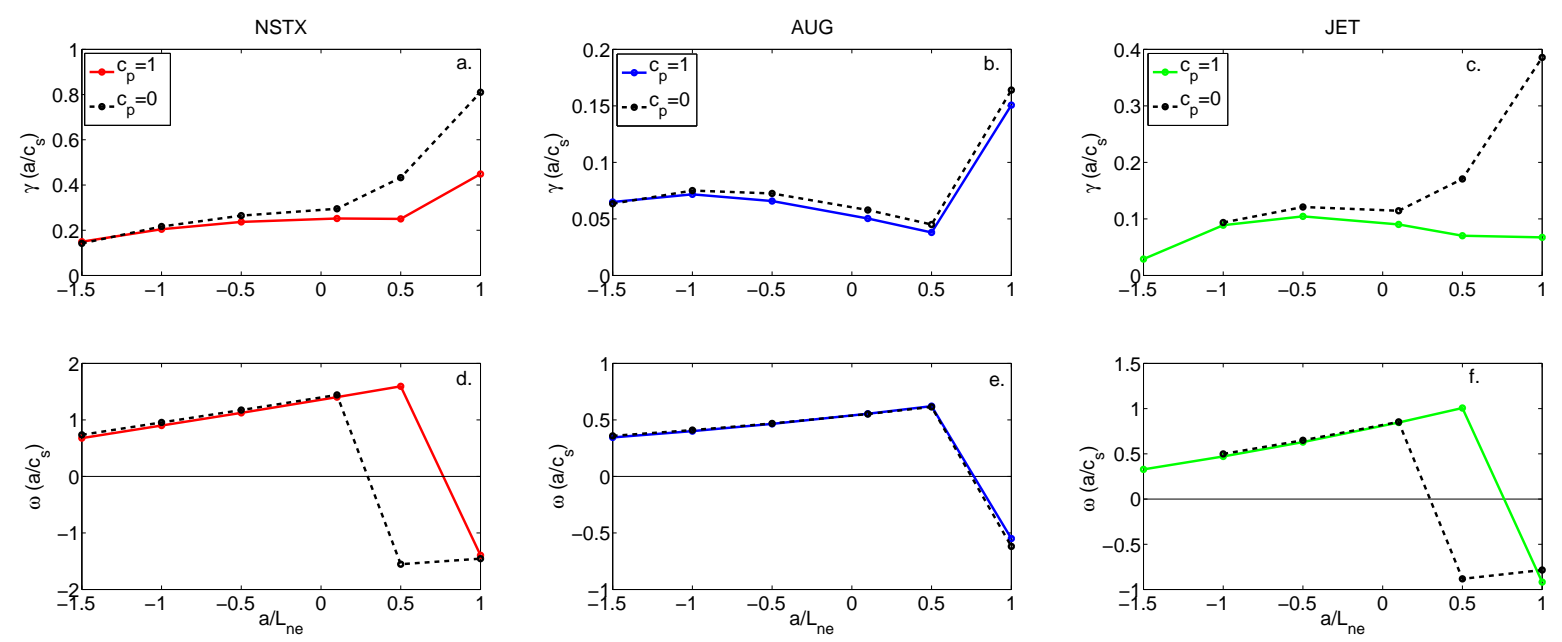

FIG. 9: Imaginary and real parts of eigenvalues $\left(\gamma, \omega_{r}\right)$ as functions of $a / L_{n e}$ with $c_{p}=1$ and $c_{p}=0$. Red solid lines: NSTX, blue dashed: ASDEX-UG, green dash-dotted: JET.

\section{CONCLUSIONS}

We have investigated the onset and parametric dependences of the MTM instability in the core $\left(\rho_{\text {tor }}=0.6\right)$ of a spherical (NSTX), and two conventional tokamaks (ASDEX-UG, JET). The quasilinear transport is computed using the gyrokinetic code GYRO in the flux-tube (local) limit. In confirmation with previous studies, we found that for the experimentally relevant plasma parameters the MTMs are linearly the dominant instability in NSTX and ASDEX-UG. Under typical JET baseline parameters considered here the MTMs are also found as the dominant linear instability. In NSTX and JET the maximum of the MTM linear growth rate is located at higher mode numbers than that for ASDEX-UG. Therefore, the previously discussed idea that the higher mode number MTMs are the characteristics of the spherical tokamaks, while lower mode number MTMs are the characteristics of the conventional tokamaks is not supported by our results.

Parametric scaling of the MTM instability in the core of these plasmas, revealed that a finite level of $\nu_{e i}$ and $\beta_{e}$ are needed in order for MTMs to become unstable, and when 
unstable, they can remain the dominant instability over a wide range in $\nu_{e i}$ and $\beta_{e}$. The linear MTM growth rate seems to be only weakly dependent on $\beta_{e}$, and exhibits a weak but non-monotonic dependence on collisionality, $\nu_{e i}$. By neglecting the collisionality or $\beta_{e}$ effects, the ITG/TEM modes appear as the most unstable modes in all three studied machines.

A strong dependence for the growth rate of MTMs on electron temperature gradient is found in NSTX, while for ASDEX-UG and JET the MTM growth rate is found to be less sensitive to this parameter. The MTM growth rate significantly increases as $a / L_{T e}$ increases in NSTX, but in ASDEX-UG and JET a weak and non-monotonic dependence on $a / L_{T e}$ is found. These results indicate that while $a / L_{T e}$ is a fundamental drive for the MTMs in these plasmas, it can contribute to the stabilization of the mode as well; this non-trivial behavior is more pronounced in the ASDEX-UG and JET plasmas.

Similar trends are observed in all three machines when scanning for the electron density gradient, where the MTMs linear growth rates show again a non-monotonic dependences on $a / L_{n e}$ parameter with peaks located in the negative $a / L_{n e}$ region corresponding to slightly hollow electron density profiles.

We have investigated the impact of a finite $\alpha_{M H D}$ on the onset and characteristics of MTM instability in various parametric scans, and we have observed that the stabilization of $\alpha_{M H D}$ parameter on the MTM mode itself is not significant in all machines. However, its impact on the ITG/TEM unstable modes can result in a strong suppression of these modes allowing for the MTM to remain the dominant instability for a wider range in considered parameters, i.e. $\beta_{e}, a / L_{T e}$ and $a / L_{n e}$. The non-monotonic and weaker dependence of the MTM on the electron temperature/density gradient however, can not be explained by $\alpha_{M H D}$ effects.

In the studied cases the MTM drives mostly electron heat transport through $\delta A_{\|}$fluctuations, while other transport channels and contributions from $\delta \phi$ and $\delta B_{\|}$are significantly smaller. For the ion heat flux and particle fluxes, the transport driven by MTMs are negligible, however, if ballooning modes like ITG/TEM/KBM are also present, even as subdominant modes, these fluxes are mainly driven by these modes and therefore the overall ion heat and particle transport may not be negligible. However, these findings are based on linear analysis and further non-linear studies are needed for their confirmation.

Finally, we would like to stress that the cases which we have considered have been chosen because they were unstable to MTMs. However, particularly large aspect ratio tokamaks, 
being unstable to MTMs should not be considered a generic property.

\section{Acknowledgments}

The authors would like to thank F Jenko, J Candy and C Angioni for valuable comments, and J Candy for providing the GYRO code. This work was funded by the European Communities under Association Contract between EURATOM and Vetenskapsrådet. The views and opinions expressed herein do not necessarily reflect those of the European Commission.

\section{Reference}

[1] M. Shimada, D. J. Campbell, V. Mukhovatov, M. Fujiwara, N. Kirneva, K. Lackner, M. Nagami, V. D. Pustovitov, N. Uckan and J. Wesley International Tokamak Physics Activity Topical Group Chairs, Cochairs and Chapter Coordinators: N. Asakura, A. E. Costley, A. J. H. Donné, E. J. Doyle, A. Fasoli, C. Gormezano, Y. Gribov, O. Gruber, T. C. Hender, W. Houlberg, S. Ide, Y. Kamada, A. Leonard, B. Lipschultz, A. Loarte, K. Miyamoto, V. Mukhovatov, T. H. Osborne, A. Polevoi and A. C. C. Sips, Nucl. Fusion 47, S1 (2007).

[2] D. C. McDonald, L. Laborde, J. C. DeBoo, F. Ryter, M. Brix, C. D. Challis, P. de Vries, C. Giroud, J. Hobrik, D. Howell, E. Joffrin, T. C. Luce, J. Mailloux, V. Pericoli-Ridolfini, A. C. C. Sips, K. Thomsen and JET EFDA Contributors, Plasma Phys. Control. Fusion 50, 124013 (2008).

[3] R. E. Waltz and R.L. Miller, Phys. Plasmas 6, 4265 (1999).

[4] D. J. Applegate, C. M. Roach, J. W. Connor, S. C. Cowley, W. Dorland, R. J. Hastie and N. Joiner, Plasma Phys. Control. Fusion 49, 1113 (2007).

[5] W. Guttenfelder, J. Candy, S. M. Kaye, W. M. Nevins, E. Wang, R. E. Bell, G. W. Hammett, B. P. LeBlanc, D. R. Mikkelsen and H. Yuh, Phys. Rev. Lett. 106, 155004 (2011).

[6] H. Doerk, F. Jenko, M. J. Pueschel and D. R. Hatch, Phys. Rev. Lett. 106, 155003 (2011).

[7] D. Told, F. Jenko, P. Xanthopoulos, L. D. Horton and E. Wolfrum, Phys. of Plasmas 15, 102306 (2008). 
[8] J. F. Drake and Y. C. Lee, Phys. Fluids 20, 1341 (1977).

[9] P. J. Catto and M. N. Rosenbluth, Phys. Fluids 24, 243 (1981).

[10] J. W. Connor, S. C. Cowley and R. J. Hastie, Plasma Phys. Control. Fusion 32, 799 (1990).

[11] D. Dickinson, C. M. Roach, S. Saarelma, R. Scannell, A. Kirk and H. R. Wilson, arXiv:1209.3695v1 (2012).

[12] W. Guttenfelder, J. Candy, S. M. Nevins, R. E. Bell, G. W. Hammett, B. P. LeBlanc and H. Yuh, Phys. of Plasmas 19, 022506 (2012).

[13] W. Guttenfelder, J. Candy, S. M. Kaye, W. M. Nevins, E. Wang, J. Zhang, R. E. Bell, N. A. Crocker, G. W. Hammett, B. P. LeBlanc, D. R. Mikkelsen, Y. Ren and H. Yuh, Phys. of Plasmas 19, 056119 (2012).

[14] H. Doerk, F. Jenko, T. Görler, D. Told, M. J. Pueschel and D. R. Hatch, Phys. of Plasmas 19, 055907 (2012).

[15] B. V. Chirikov, Phys. Rep. 52, 263 (1979).

[16] J. Candy and R. E. Waltz, J. Comput. Phys., 186, 545 (2003).

[17] J. Candy, Plasma Phys. Control. Fusion, 51, 105009 (2009).

[18] E. A. Belli and J. Candy, Phys. Plasmas, 17, 112314 (2010). 